\title{
Atribuições de Causalidade pela Infecção por HIV
}

\author{
Fernando Martins de Azevedo* \\ Universidade Federal do Rio Grande do Sul - UFRGS, Porto Alegre, RS, Brasil \\ ORCID: https://orcid.org/0000-0002-2155-5975 \\ Damião Soares de Almeida Segundo** \\ Universidade Federal do Rio Grande do Sul - UFRGS, Porto Alegre, RS, Brasil \\ ORCID: https://orcid.org/0000-0003-2407-0583 \\ Marina Feijó ${ }^{* * * *}$ \\ Pontifícia Universidade Católica do Rio Grande do Sul - PUCRS, Porto Alegre, RS, Brasil \\ ORCID: https://orcid.org/0000-0002-6031-9780 \\ Henrique Caetano Nardi $* * * *$ \\ Universidade Federal do Rio Grande do Sul - UFRGS, Porto Alegre, RS, Brasil \\ ORCID: https://orcid.org/0000-0001-6058-1642 \\ Angelo Brandelli Costa****** \\ Pontifícia Universidade Católica do Rio Grande do Sul - PUCRS, Porto Alegre, RS, Brasil \\ ORCID: https://orcid.org/0000-0002-0742-8152
}

\begin{abstract}
RESUMO
A prevalência de HIV acomete significativamente a população brasileira. Os homens que fazem sexo com homens (HSH) e as mulheres trans são afetados desproporcionalmente pela epidemia. Evidências indicam maiores atribuições de culpa, controle e responsabilidade para pessoas que se infectam por HIV em situações de sexo desprotegido, especialmente homossexuais. Portanto, o presente estudo experimental buscou testar de que forma estudantes e profissionais da área da saúde atribuem culpa, controle e responsabilidade pela infecção por HIV. A amostra foi composta por estudantes e profissionais do município de Porto Alegre. As/os participantes do estudo foram alocadas/os randomicamente em seis cenários, que mostravam uma infecção por HIV via sexo desprotegido ou via transfusão de sangue, sendo que a identidade da pessoa da cena varia entre homem heterossexual, homem homossexual e mulher trans. Os resultados indicaram maiores atribuições de controle, culpa e responsabilidade para o homem homossexual e para a mulher trans, somente no cenário "sexo desprotegido".
\end{abstract}

Palavras-chave: HIV, estigma social, preconceito, psicologia social.

\section{Attributions of Causality for HIV Infection}

\begin{abstract}
The HIV epidemic is a significant problem in Brazil. Men who have sex with men (MSM) and transgender women are disproportionately affected by the epidemic. Evidence shows that attributions of onset controllability, blame, and responsibility for the HIV infection are higher
\end{abstract}

ISSN 1808-4281 
in cases of unprotected sex and also when the person is homosexual. This article presents the results of an experimental study, which tested the impact of the attributions of onset controllability, blame, and responsibility for the HIV infection when a person is homosexual or transgender. The participants were allocated randomly in six experimental scenarios. The scenarios were about an HIV infection by unprotected sex or by blood transfusion. The infected patient could be a heterosexual man, homosexual man or transgender woman. Our results indicated higher attributions of onset controllability, blame, and responsibility to the homosexual man and to the transgender woman, only in cases of infection by unprotected sex.

Keywords: HIV, social stigma, prejudice, social psychology.

\section{Atribuciones de Causalidad por Infección con VIH}

\section{RESUMEN}

La epidemia de VIH acomete significativamente a la población brasileña. Los hombres que tienen sexo con hombres (HSH) y las mujeres transgénero son afectados desproporcionadamente por la epidemia. Evidencias indican mayores atribuciones de culpa, control y responsabilidad para personas que se infectan por el VIH en situaciones de sexo desprotegido, especialmente homosexuales. Así, este artículo presenta y discute los resultados de un estudio experimental que buscó testar cómo las personas atribuyen culpa, control y responsabilidad por la infección por VIH. La muestra fue compuesta por estudiantes y profesionales del municipio de Porto Alegre. Las/Los participantes fueron asignadas/os al azar en seis escenarios, que mostraban una infección por VIH a través del sexo desprotegido o transfusión de sangre, con la identidad de la persona de la escena que varía entre hombre heterosexual, hombre homosexual y mujer trans. Los resultados indicaron mayores atribuciones de control, culpa y responsabilidad para el hombre homosexual y para la mujer trans, solamente en los escenarios "sexo sin protección".

Palabras clave: VIH, estigma social, prejuicio, psicología social.

A epidemia mundial de HIV acomete significativamente a população brasileira. Segundo o último boletim epidemiológico, foram notificados 300496 casos de pessoas vivendo com HIV no país, de 2007 a 2018. Destes, 69\% são homens e 31\% são mulheres. (Ministério da Saúde, 2018). A cidade de Porto Alegre, no entanto, apresentou, no ano de 2018, a terceira maior taxa de detecção de aids entre as capitais brasileiras: 53,7 casos para cada 100 mil habitantes. Historicamente, todavia, a capital gaúcha tem sido a líder nacional de detecção de aids entre as capitais, chegando a ter mais de 100 casos para cada 100 mil habitantes. Apesar da recente diminuição, a taxa porto-alegrense ainda é o dobro da média estadual e com índice três vezes maior do que o nacional (Ministério da Saúde, 2018). 
Fernando Martins de Azevedo, Damião Soares de Almeida Segundo, Marina Feijó, Henrique Caetano Nardi, Angelo Brandelli Costa

Os homens que fazem sexo com homens (HSH) e as mulheres trans são notadamente afetados pela epidemia. No Brasil, nesses dois grupos, a prevalência de HIV é alta e continua aumentando. No ano de 2007, dentre os casos notificados de HIV em indivíduos do sexo masculino (acima de 13 anos e que se infectaram pela via sexual), 37,2\% estavam na categoria de exposição homossexual ou bissexual. Em 2019, no entanto, esse percentual é de 55\%. (Ministério da Saúde, 2018). Em Porto Alegre, estima-se que a prevalência de HIV em HSHs que conhecem sua condição sorológica seja de 4,1\% (Melo, 2006).

Ademais, as mulheres trans vêm apresentando a maior vulnerabilidade frente à epidemia. Segundo uma meta-análise, este grupo em questão possui 48,8 vezes mais chances de infecção por HIV do que a população geral (Baral et al., 2013). No Rio Grande do Sul, esse índice é ainda maior, apontando uma chance 55,55 vezes maior de contrair HIV, estando nesse segmento populacional. (Costa et al., 2015). Tais evidências não ocorrem por acaso, visto que são fatores sociais e políticos que provocam a vulnerabilização (Costa, 2015).

Os principais fatores que vulnerabilizam as mulheres trans e os homens que fazem sexo com homens para a infeção por HIV são o preconceito e a discriminação, seja pela dificuldade de acesso aos serviços de saúde ou pela vivência direta do preconceito (Poteat et al., 2015). O preconceito é expresso na forma de atitudes adversas ou hostis em relação a alguém que pertence a um determinado grupo, simplesmente pelo fato de pertencer a esse grupo (Allport, 1954), o preconceito atinge significativamente sujeitos que pertencem a grupos minoritários. Ao sofrerem preconceito em serviços públicos de saúde, por exemplo, homens que fazem sexo com homens (HSH) e mulheres trans podem passar a evitar tais serviços, devido à expectativa de discriminação (Meyer, 1995). Essa tendência aparece em estudo de Costa et al. (2018), realizado com pessoas trans e em não conformidade de gênero. Nessa amostra, 6,5\% dos respondentes disseram que evitavam a testagem de HIV por terem medo de vivenciar preconceito ou discriminação.

Dado este cenário, ao enfrentar essas diversas formas de discriminação e sofrimento, os indivíduos ficam mais vulneráveis ao adoecimento, inclusive por HIV/AIDS. A vulnerabilidade para a infecção está relacionada à situação de isolamento, preconceito e estigma, não advindo diretamente da sexualidade ou gênero das pessoas (Costa, 2015). Uma dessas manifestações de preconceito envolve o processo de atribuição de causalidade. Tratase de julgamentos realizados sobre si mesmo/a e/ou sobre os/as outros/as, através dos quais buscamos explicações para os acontecimentos cotidianos (McDonell, 1993). As atribuições ocorrem porque as pessoas desejam conhecer a fonte de suas experiências, saber de onde 
vieram e como surgiram, para assim serem capazes de controlar eventos futuros (Heider, 1970).

A atribuição acerca do porquê um fenômeno ocorre pode ser denominada atribuição de causalidade. Quando as pessoas atribuem a causalidade de um fenômeno a forças do ambiente e causas situacionais, pode-se dizer que se trata de atribuição externa. O contrário acontece quando a causalidade de algo é atribuída a características individuais das pessoas, o que se pode chamar de atribuição interna. Portanto, atribuição interna reflete a responsabilidade individual para um evento que poderia ser controlado, enquanto a externa indica a responsabilidade do ambiente para um evento que não poderia ser controlado (Nasser, Singhal, \& Abouchedid, 2005). Em geral, atribuições também são acompanhadas de uma resposta emocional e comportamental (Weiner, 1995).

Quando se atribui responsabilidade individual (atribuições internas) para a infecção com o vírus HIV, os resultados obtidos geralmente são respostas emocionais de raiva e comportamentos negativos (menor intenção em ajudar). Por outro lado, atribuições externas (quando se atribui responsabilidade ao ambiente), geralmente resultam em reações emocionais e comportamentais positivas, como simpatia para com a pessoa. É comum, no caso das infecções por HIV, que atribuições internas sejam feitas nos casos de infecção via sexo desprotegido. Em infecções via transfusão de sangue, costuma-se observar atribuições externas (Mantler, Schellenberg, \& Page, 2003). As atribuições, no entanto, não necessariamente têm relação direta com a explicação real do fenômeno, mas são na verdade um processo no qual, a partir das experiências, um indivíduo busca compreender a causa de um fenômeno (Dela Coleta \& Dela Coleta, 2006). A infecção por HIV, por exemplo, é um processo multideterminado cujas causas muitas vezes escapam à percepção dos indivíduos. Logo, é possível que as pessoas façam atribuições internas quando há uma situação de infecção (Seacat, Hirschman, \& Mickelson, 2007), sem considerar o fato de que a vulnerabilidade para a epidemia se dá por um conjunto de fatores biológicos, psicológicos, sociais e políticos (Ayres, Paiva, \& França Junior, 2011).

Considerando os problemas decorrentes do processo de atribuição, Seacat et al. (2007) realizaram um estudo experimental para medir as atribuições de controle, responsabilidade e culpa de estudantes de psicologia em relação a quatro situações experimentais. Os cenários foram os seguintes: heterossexual se infecta com HIV numa situação de sexo desprotegido (1); homossexual se infecta com HIV numa situação de sexo desprotegido (2); heterossexual se infecta com HIV numa transfusão de sangue (3); e homossexual se infecta com HIV numa transfusão de sangue (4). Nesse estudo, as/os participantes atribuíram consideravelmente mais 
controle, responsabilidade e culpa nos cenários que envolviam sexo desprotegido. Ainda nesses cenários, raiva foi significativamente mais reportada do que simpatia, bem como a intenção de ajudar foi bem menor em comparação a cenários de infecção via transfusão de sangue. Esse estudo também analisou o papel da orientação sexual nas atribuições de controle, responsabilidade e culpa. Em ambos os cenários experimentais, participantes atribuíram mais controle, mais culpa e mais responsabilidade aos homossexuais do que aos heterossexuais.

Ademais, existem outros estudos que utilizam este mesmo modelo de atribuição de causalidade, inclusive com outras doenças que não HIV. Em uma revisão sistemática sobre estigma relacionado à obesidade, os resultados indicaram que quando a causa da obesidade é atribuída ao controle pessoal do indivíduo, maior a probabilidade de comportamento estigmatizante (Sikorski et al., 2011). Em outra revisão sistemática, que analisou as atribuições da causalidade sobre doenças cardíacas, foi identificado que a maioria das/os pacientes justificava o seu adoecimento como sendo causado pelo destino e pelo azar. Uma possível explicação para essa atribuição, segundo os autores, seria o desejo das/os pacientes de não se sentirem responsáveis pelo adoecimento, visto que quanto mais responsabilidade fosse atribuída a elas/es, menos as/os profissionais de saúde estariam dispostos a ajudar (French, Senior, Weinman, \& Marteau, 2001).

Quando as/os profissionais de saúde atribuem significativamente mais controle, culpa e responsabilidade para os homossexuais do que para os heterossexuais, isso é acompanhado por maiores reações emocionais de raiva e menor intenção de ajudar (Seacat et al., 2007). As atribuições, as reações emocionais de raiva e a menor disposição para ajudar podem acarretar em vivência direta de discriminação, o que pode levar à frequente evitação dos serviços de saúde por parte de usuárias/os homossexuais (Meyer, 1995). A evitação dos serviços de saúde torna essa população vulnerável à epidemia de HIV/AIDS (Costa et al., 2018).

Neste mesmo sentido, pode-se dizer que as pessoas trans são ainda mais vulnerabilizadas (Safer et al., 2016). Processos de discriminação por parte das/os profissionais de saúde causam um impacto significativo no acesso de pessoas trans a serviços de saúde (Bockting, Robinson, Benner, \& Scheltema, 2004). Além de serem extremamente vulnerabilizadas para a epidemia de HIV/AIDS no Brasil, mulheres transexuais são desproporcionalmente afetadas pela epidemia no mundo todo, sendo a taxa de prevalência nessa população cerca de $20 \%$ em níveis mundiais (Baral et al., 2013).

Em razão disso, propomos um estudo experimental, em formato semelhante ao realizado por Seacat et al. (2007), porém incluindo cenários com mulheres trans. O objetivo 
geral deste estudo é identificar mudanças nos processos de atribuição de causalidade pela infecção por HIV em uma amostra de estudantes universitários da área da saúde e profissionais da saúde do município de Porto Alegre. Ainda, busca-se analisar a relação entre as formas de atribuição de causalidade, a intenção de ajudar e as reações emocionais (objetivo específico 1), bem como comparar as atribuições de controle, responsabilidade e culpa, as reações emocionais e a intenção de ajudar usuários heterossexuais, homossexuais e mulheres trans em cenários de infecção via transfusão de sangue e de infecção via sexo sem o uso de preservativo (objetivo específico 2).

As hipóteses deste estudo são de que há uma relação direta entre os três tipos de atribuição (culpa, controle e responsabilidade); de que há uma relação inversa entre as reações emocionais (raiva, simpatia); e de que a reação de raiva se relaciona diretamente com as atribuições. Além disso, espera-se que a intenção de ajudar se relacione inversamente com as atribuições e a reação de raiva e diretamente com a simpatia. Ademais, trabalhamos com a hipótese de que as/os participantes irão atribuir mais culpa, controle e responsabilidade para a usuária trans e para o usuário homossexual em relação ao usuário heterossexual, tanto no cenário transfusão de sangue quanto no cenário "sexo desprotegido". Espera-se também maiores índices de reações emocionais de raiva, menores de simpatia e menores de intenção de ajudar em relação à usuária trans e ao usuário homossexual em comparação com o usuário heterossexual.

\section{Método}

O presente estudo utilizou a vinheta experimental do estudo de Seacat et al. (2007), que foi adaptada e traduzida para o português brasileiro. Trata-se, portanto de um estudo experimental de delineamento fatorial $3 \times 2$.

\section{Participantes}

O estudo abrangeu um total de 340 pessoas que acessaram o questionário na plataforma qualtrics e, portanto, eram elegíveis para amostra final. No entanto, duas pessoas foram excluídas por não terem consentido ao estudo $(n=2)$, outras oitenta e três por terem deixado protocolos incompletos $(n=83)$ e quarenta e nove pessoas foram excluídas por não terem respondido corretamente às perguntas de checagem $(n=49)$. Depois de aplicados os 
Fernando Martins de Azevedo, Damião Soares de Almeida Segundo, Marina Feijó, Henrique Caetano Nardi, Angelo Brandelli Costa

critérios de exclusão, a amostra final do estudo chegou a 206 participantes $(n=206)$. Os 206 foram alocados nos seis cenários conforme a Figura 1:

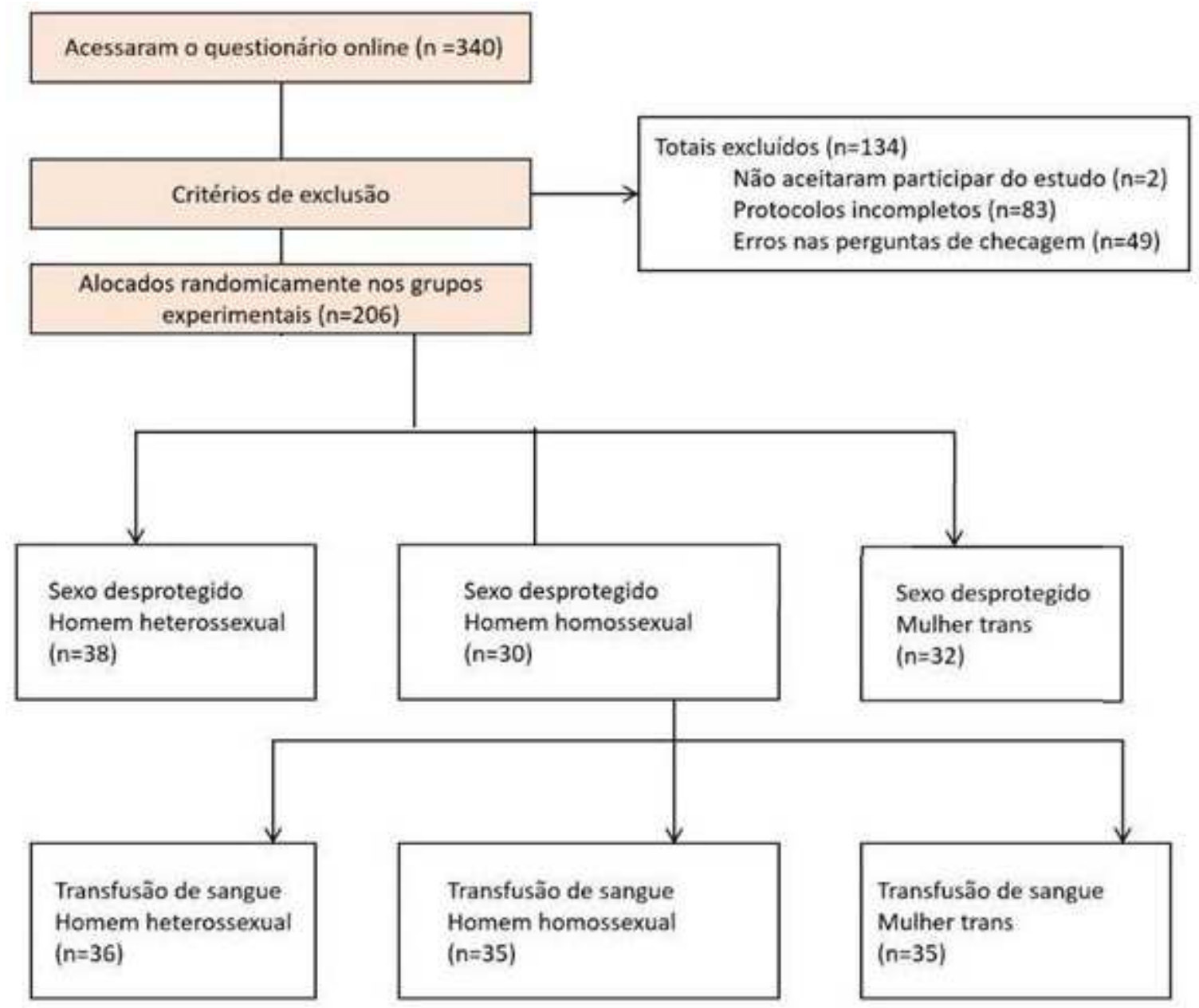

Figura 1. Participantes do estudo.

Os dados sociodemográficos do estudo estão demonstrados na Tabela 1. 
Fernando Martins de Azevedo, Damião Soares de Almeida Segundo, Marina Feijó, Henrique Caetano Nardi, Angelo Brandelli Costa

Tabela 1

Características sociodemográficas da amostra

\begin{tabular}{lrr}
\hline Profissional ou Estudante* & $\mathrm{n}$ & $\%$ \\
$\quad$ Profissional da área & 31 & 15 \\
Estudante de graduação & 182 & 88,35 \\
& & \\
Gênero & $\mathrm{n}$ & $\%$ \\
Mulher & 180 & 87,38 \\
Homem & 26 & 12,62 \\
& & \\
Orientação sexual & $\mathrm{n}$ & $\%$ \\
Heterossexual & 140 & 67,96 \\
Homossexual & 18 & 8,74 \\
Bissexual & 40 & 19,42 \\
Não sabe & 3 & 1,46 \\
Outra & 5 & 2,43 \\
& & \\
Religião & $\mathrm{n}$ & $\%$ \\
Possui religião & 95 & 46,12 \\
Não possui religião & 111 & 53,88 \\
\hline
\end{tabular}

*Múltiplas respostas

\section{Instrumentos}

Sociodemográficas. As/os participantes responderam a perguntas sociodemográficas sobre: gênero, idade, orientação sexual, profissão e religiosidade.

Atribuição de controle, culpa e responsabilidade. A escala que mediu as atribuições que as/os participantes fizeram em relação à vinheta possuía um total de 13 itens (e.g., "A pessoa da cena é responsável pelo seu adoecimento"; Mantler et al., 2003), respondidos por meio de uma escala Likert de cinco pontos. As respostas podiam variar entre "concordo totalmente" e "discordo totalmente". Os itens avaliam o quanto o participante atribuiu à pessoa da cena responsabilidade pelo o adoecimento, possibilidade de controle, e culpabilização pelo adoecimento. O estudo de Seacat et al. (2007) avaliou a consistência de cada uma das subescalas e encontrou alfas aceitáveis: responsabilidade, $\alpha=0,91$; controle, $\alpha=0,88$; e culpa, $\alpha=0,84$.

Reações emocionais. Também desenvolvido por Mantler et al. (2003), a escala sobre reações emocionais possui oito itens. Desses, quatro correspondem a quanto o participante sente raiva da pessoa da cena (e.g. "Eu sinto raiva da pessoa da cena") e outros quatro a quanto sentem de simpatia (e. g. "Eu sinto compaixão pela pessoa da cena"). Sendo 
respondidos por meio de escala Likert de 5 pontos. As respostas podiam variar entre "concordo totalmente" e "discordo totalmente". Esta escala possui consistência interna aceitável, $\alpha=0,71$ (Seacat et al., 2007)

Intenção de ajudar. A intenção de ajudar o/a usuário/a foi avaliada por uma escala desenvolvida por Dooley (1995) que contém 10 itens (e.g. "Ajudaria a pessoa da cena a caminhar", "Iria à farmácia pegar uma receita para a pessoa da cena") que avaliam especificamente a intenção de ajudar pessoas vivendo com HIV ou AIDS (PVHA) por meio de uma escala Likert de cinco pontos. As respostas podiam variar entre "concordo totalmente" e "discordo totalmente". A escala possui consistência interna de $\alpha=0,88$ (Seacat et al., 2007).

\section{Procedimentos}

\section{Coleta de Dados}

As/os participantes foram estudantes de graduação dos cursos de Medicina, Enfermagem, Odontologia, Psicologia e Educação Física da Universidade Federal do Rio Grande do Sul (UFRGS) e profissionais da saúde do município de Porto Alegre. No caso dos estudantes, a Pró-Reitoria de Graduação da UFRGS, através das suas coordenadorias de curso, disparou e-mails para os estudantes convidando-os para participar. O mesmo foi feito pelas Gerências Distritais da Secretaria Municipal de Saúde de Porto Alegre, aos servidores da área da saúde. O projeto foi aprovado pelo Comitê de Ética em Pesquisa (CEP) da UFRGS e pelo Comitê de Ética em Pesquisa da Secretaria Municipal de Saúde de Porto Alegre (CEP SMSPA). Todas/os participantes responderam ao Termo de Consentimento Livre e Esclarecido (TCLE).

Ao consentir em participar da pesquisa, a/o participante foi randomicamente alocada/o em um dos seis cenários experimentais. Após ler a vinheta, as/os participantes responderam duas perguntas de checagem (para verificar se compreenderam a vinheta): "qual orientação sexual e identidade de gênero da pessoa da cena?" e "como a pessoa da cena se infectou com HIV?". Participantes que não responderam corretamente a essas perguntas foram excluídos da amostra final. A vinheta contém uma situação de infecção por HIV. A pessoa da cena é um homem heterossexual, um homem homossexual ou uma mulher trans e a infecção ocorreu necessariamente em uma situação de sexo desprotegido ou via transfusão de sangue. Os seis cenários possíveis são: homem heterossexual, sexo desprotegido (1); homem heterossexual, transfusão de sangue (2); homem homossexual, sexo desprotegido (3); homem homossexual, 
transfusão de sangue (4); mulher trans, sexo desprotegido (5) e mulher trans, transfusão de sangue (6). Os cenários com homem heterossexual podem ser considerados grupo controle enquanto os demais são os grupos experimentais.

\section{Análise de Dados}

Para alcançar o primeiro objetivo específico foi realizada uma correlação linear com o intuito de identificar a relação entre as atribuições de causalidade (culpa, controle e responsabilidade), as reações emocionais (raiva e simpatia) e a intenção de ajudar. Posteriormente, para alcançar o segundo objetivo específico, foram realizadas ANOVAs para comparar as variáveis entre os diferentes grupos identitários (homem homossexual, mulher trans, homem heterossexual) para os distintos cenários de infecção (sexo desprotegido e transfusão de sangue).

\section{Resultados}

A Tabela 2 traz os resultados da correlação entre as atribuições de causalidade (controle, culpa e responsabilidade), intenção de ajudar e reações emocionais (raiva, simpatia). Ao verificar as correlações, percebe-se que as atribuições de controle, culpa e responsabilidade estão fortemente correlacionadas entre si. Isso significa que se a pessoa da cena é entendida como responsável pelo que lhe aconteceu, as atribuições de culpa e controle devem variar no mesmo sentido. Verifica-se também que a intenção de ajudar não possui correlação significativa com a maioria das variáveis, com exceção de simpatia. Raiva, no entanto, possui correlação positiva significativa com culpa, controle e responsabilidade, enquanto simpatia possui correlação negativa - também significativa - com as mesmas variáveis. 
Fernando Martins de Azevedo, Damião Soares de Almeida Segundo, Marina Feijó, Henrique Caetano Nardi, Angelo Brandelli Costa

Tabela 2

Correlação entre Atribuições de Causalidade, Intenção de Ajudar e Reações Emocionais

\begin{tabular}{lllllll}
\hline Medida & 1 & 2 & 3 & 4 & 5 & 6 \\
\hline 1. Controle & - & & & & & \\
2. Culpa & $0,81^{*}$ & - & & & & \\
3. Responsabilidade & $0,88^{*}$ & $0,82^{*}$ & - & & & \\
4. Intenção de ajudar & 0,002 & $-0,09$ & $-0,06$ & - & & \\
5. Raiva & $0,23^{*}$ & $0,29 *$ & $0,19^{*}$ & 0,05 & - & \\
6. Simpatia & $-0,35^{*}$ & $-0,42^{*}$ & $-0,37^{*}$ & $-0,19^{*}$ & $-0,11$ & - \\
\hline
\end{tabular}

${ }^{*} p<0,05 *{ }^{* *} p<0,01$

Os resultados das ANOVAs são apresentados nas tabelas 3 e 4. No cenário sangue, perceber-se que houve uma diferença estatisticamente significativa apenas na variável responsabilidade.

Tabela 3

ANOVA Comparando Atribuições de Causalidade, Intenção de Ajudar e Reações Emocionais para os Grupos Heterossexual, Homossexual e Transexual no Cenário Sangue

\begin{tabular}{|c|c|c|c|c|c|c|c|}
\hline & \multicolumn{2}{|c|}{ Heterossexual } & \multicolumn{2}{|c|}{ Homossexual } & \multicolumn{2}{|c|}{ Transexual } & \multirow[b]{2}{*}{$\mathrm{F}$} \\
\hline & $\mathrm{M}$ & DP & $\mathrm{M}$ & DP & $\mathrm{M}$ & DP & \\
\hline Controle & 18,16 & 2,70 & 17,97 & 3,09 & 18,61 & 2,38 & 0,50 \\
\hline Culpa & 19,16 & 1,79 & 18,71 & 2,12 & 19,70 & 1,11 & 2,82 \\
\hline Responsabilidade & 19,27 & 1,48 & 17,80 & 2,93 & 19,35 & 1,51 & $6,12 *$ \\
\hline Intenção de ajudar & 14,44 & 7,51 & 15,22 & 7,56 & 12,85 & 4,11 & 1,14 \\
\hline Raiva & 17,50 & 2,64 & 17,14 & 2,64 & 17,23 & 2,78 & 0,16 \\
\hline Simpatia & 5,27 & 1,75 & 5,82 & 2,3 & 4,85 & 1,95 & 2,03 \\
\hline
\end{tabular}

$* p<0,01 * * p<0,05$

Tabela 4

ANOVA Comparando Atribuições de Causalidade, Intenção de Ajudar e Reações Emocionais Para os Grupos Heterossexual, Homossexual e Transexual no Cenário Sexo

\begin{tabular}{|c|c|c|c|c|c|c|c|}
\hline & \multicolumn{2}{|c|}{ Heterossexual } & \multicolumn{2}{|c|}{ Homossexual } & \multicolumn{2}{|c|}{ Transexual } & \multirow[b]{2}{*}{$\mathrm{F}$} \\
\hline & $\mathrm{M}$ & $\mathrm{DP}$ & $\mathrm{M}$ & $\mathrm{DP}$ & $\mathrm{M}$ & DP & \\
\hline Controle & 6,34 & 2,31 & 8,26 & 2,87 & 8,87 & 3,50 & $7,38^{*}$ \\
\hline Culpa & 10,81 & 3,08 & 13,80 & 3,49 & 13,48 & 4,28 & $7,19 *$ \\
\hline Responsabilidade & 8,31 & 2,57 & 10,40 & 3,22 & 10,12 & 3,80 & $4,37^{* *}$ \\
\hline Intenção de ajudar & 14,63 & 7,53 & 13,50 & 6,74 & 14,00 & 6,59 & 0,22 \\
\hline Raiva & 15,81 & 3,44 & 16,73 & 3,18 & 16,63 & 3,19 & 0,82 \\
\hline Simpatia & 7,50 & 2,65 & 5,96 & 2,14 & 6,33 & 2,28 & $3,90 * *$ \\
\hline
\end{tabular}

$* p<0,01 * * p<0,05$ 
Fernando Martins de Azevedo, Damião Soares de Almeida Segundo, Marina Feijó, Henrique Caetano Nardi, Angelo Brandelli Costa

Em razão do resultado dessa ANOVA, foram realizados testes post-hoc Tuckey HSD para a variável responsabilidade no cenário sangue; o grupo Homossexual $(\mathrm{M}=17,80$, $\mathrm{DP}=2,93)$ se diferenciou significativamente do Heterossexual $(\mathrm{M}=19,27, \mathrm{DP}=1,48 ; p<0,01) \mathrm{e}$ do Transexual $(\mathrm{M}=19,35, \mathrm{DP}=1,51 ; p<0,01)$.

Por fim, no cenário sexo desprotegido, todas as variáveis tiveram diferenças estatisticamente significativa com exceção da raiva e da intenção de ajudar. Testes post-hoc Tuckey HSD indicaram diferenças significativas entre o grupo Heterossexual e o Homossexual para controle $(\mathrm{M}=6,24, \mathrm{DP}=2,31 ; \mathrm{M}=8,26, \mathrm{DP}=2,87 ; p<0,05)$, culpa $(\mathrm{M}=10,81, \mathrm{DP}=3,08 ; \mathrm{M}=13,80, \mathrm{DP}=3,49 ; p<0,01)$, responsabilidade $(\mathrm{M}=8,31, \mathrm{DP}=2,57$; $\mathrm{M}=10,40, \mathrm{DP}=3,22 ; p<0,05)$ e simpatia $(\mathrm{M}=7,50, \mathrm{DP}=2,65 ; \mathrm{M}=5,96, \mathrm{DP}=2,14 ; p<0,05)$; e entre o grupo Heterossexual e o Transsexual para controle $(\mathrm{M}=6,34, \mathrm{DP}=2,31 ; \mathrm{M}=8,87$, $\mathrm{DP}=3,50 ; \quad p<0,001)$, culpa $(\mathrm{M}=10,81, \quad \mathrm{DP}=3,08 ; \quad \mathrm{M}=13,48, \quad \mathrm{DP}=4,28 ; \quad p<0,01) \quad \mathrm{e}$ responsabilidade $(\mathrm{M}=8,31, \mathrm{DP}=2,57 ; \mathrm{M}=10,12, \mathrm{DP}=3,80 ; p<0,05)$.

\section{Discussão}

Conforme já apontado em alguns estudos internacionais (Cobb \& De Chabert, 2002; Seacat et al., 2007), foi possível perceber, uma relação significativa, direta e forte entre as atribuições de culpa, controle e responsabilidade. Por exemplo, o estudo experimental de Cobb e De Chabert (2002), era semelhante ao presente estudo, porém com vinheta única, na qual variava somente o gênero da pessoa da cena. Na vinheta, foi apresentada às/aos participantes uma situação em que uma pessoa chegava ao serviço de saúde para assistir uma aula sobre HIV/AIDS e fazer o teste de HIV, após ordem judicial. Era descrito ainda que esta pessoa havia se engajado em uma série de comportamentos que poderiam tê-la exposta à infecção. Por fim, era relatado que esta pessoa havia solicitado ajuda a um profissional de saúde para encontrar moradia, tratar o seu uso de drogas e reduzir os seus comportamentos de risco para infecção. Diante desse cenário, as/os participantes do estudo apresentaram índices elevados de atribuição de culpa e responsabilidade, bem como elevado índice de raiva e menores intenções de ajudar. Todas as variáveis tiveram correlação significativa.

Outro resultado importante a ser destacado é a relação significativa entre todas as atribuições e as reações emocionais de raiva e simpatia, de forma semelhante ao que indicou o estudo de Dooley (1995). A autora utilizou cinco cenários experimentais onde um homem descobria estar com AIDS. As formas de infecção eram: infecção por transfusão de sangue (1), infecção por sexo desprotegido numa relação heterossexual (2), infecção por sexo 
desprotegido com parceiro homossexual (3), uso de drogas injetáveis (4) ou nenhuma causa indicada (5). Após a leitura da vinheta, as/os participantes responderam questionários semelhantes aos utilizados no presente estudo. A autora relatou que a atribuição de controle foi menor no cenário sangue, resultado que também encontramos. A resposta emocional de raiva no estudo de Dooley (1995) foi significativamente maior no cenário de infecção por sexo desprotegido (hetero ou homossexual), no cenário de infecção por uso de drogas injetáveis e no cenário sem causa específica. O cenário de infecção via transfusão de sangue obteve menores índices de raiva e maiores índices de simpatia.

Os resultados do presente estudo diferem dos resultados de Dooley (1995) no que diz respeito ao tipo de parceiro sexual, posto que as nossas análises indicaram que houve maior atribuição de culpa, responsabilidade e controle ao homem homossexual e à mulher trans. No entanto, o resultado tem um aspecto similar, pois Dooley (1995) não encontrou mudanças significativas na intenção de ajudar da forma como Seacat et al. (2007) encontrou. Mesmo com a resposta emocional de raiva elevada, a amostra analisada por Dooley (1995) não teve a intenção de ajudar afetada por este fator. O presente estudo achou resultado semelhante, posto que intenção de ajudar não obteve resultado significativo em nenhum dos cenários.

Ademais, vale ressaltar que as diferenças encontradas na forma como as pessoas atribuem controle, culpa e responsabilidade foram significativas apenas no cenário de sexo. No cenário de transfusão de sangue não se pode dizer que exista essa relação. O mesmo pode ser dito sobre a reação emocional de simpatia. Em suma, no cenário onde há infecção por meio de sexo desprotegido, as pessoas atribuem mais culpa, responsabilidade e controle à mulher trans e ao homem homossexual, ao mesmo tempo em que desenvolvem mais simpatia pela pessoa heterossexual. No entanto, os resultados apresentados nesse artigo diferem de Seacat et al. (2007), que encontrou mudanças significativas na raiva e na intenção de ajudar. Esse resultado indica que apesar das/os profissionais da saúde terem um viés de atribuição mais negativo em relação à população LGBT, afirmam procurar ajudar a todos da mesma forma.

No entanto, esse viés cognitivo e atribucional é perigoso no contexto da saúde, visto que pode implicar em discriminação, mesmo que esta se manifeste através de expressões sutis. Por mais que a intenção de ajudar dos profissionais não tenha sido significativamente afetada, o fato de profissionais de atribuírem mais culpa, controle e responsabilidade aos indivíduos trans e gays pode fazer com que as pessoas sintam que estão em ambiente de estigma e preconceito nos serviços de saúde. Além disso, é possível que discursos carregados de preconceito e estigma sejam internalizados por aquelas/es que estão no serviço. Essa 
tendência foi encontrada em um estudo qualitativo realizado por Hamman, Pizzinato, Weber e Rocha (2017), que identificou nas falas das/os usuárias/os de um serviço de HIV/AIDS a presença de asserções com a presença de preconceito. Uma delas veio diretamente de um participante homossexual, que fez uma associação direta entre o fato de ser gay e engajar-se em sexo desprotegido.

A vivência do estigma relacionado ao HIV/AIDS é, segundo a literatura, algo que a aumenta a chance de desenvolvimento de uma série de desfechos negativos em saúde, incluindo: depressão, aumento do estresse emocional, ansiedade, menor índice de qualidade de vida, piores desfechos de saúde física, menor apoio social e maior índice de sexo desprotegido (Rueda et al., 2015). O preconceito, segundo Meyer (1995) pode levar a uma expectativa de discriminação, que pode fazer com que homens homossexuais e mulheres trans, considerando a amostra deste estudo, procurem menos os serviços de saúde. Ainda, existe evidência de que esse ambiente de estigma e de preconceito também possui impacto na adesão ao tratamento em pacientes que vivem com HIV (Katz et al., 2013).

Portanto, os dados nos fazem concluir que as/os profissionais de saúde e as/os estudantes de graduação dos cursos da área possivelmente não estão obtendo a formação adequada no que diz respeito às questões de gênero e sexualidade, posto que apresentam um viés negativo de atribuição para pacientes não-heterossexuais. Há de se afirmar, ainda, a possibilidade de não terem conhecimento sobre HIV/AIDS e sobre as possíveis consequências negativas que a vivência do estigma e do preconceito representam.

Por fim, ressalta-se que este estudo possui limitações. As/Os profissionais da saúde e as/os estudantes de graduação que fizeram parte da amostra estavam limitados somente aos cursos de Medicina, Enfermagem, Odontologia, Psicologia e Educação Física, sendo que a maioria eram estudantes de Enfermagem ou Psicologia. São necessários estudos com amostras maiores, que incluam profissionais de todas as áreas, como Fisioterapia, Terapia Ocupacional, Nutrição e outras áreas da saúde as quais nossa amostra não contempla. Além disso, a nossa amostra não pode ser considerada homogênea, em função de termos estudantes de graduação e profissionais da saúde (já graduados) compondo a mesma amostra. Embora esse fator não invalide os resultados, salientamos a importância da realização de estudos semelhantes que venham a comparar os dois grupos, dado que isso não foi possível com o nosso tamanho amostral. 


\section{Considerações Finais}

Os resultados apontaram a existência de uma relação entre as atribuições de causalidade e as reações emocionais, mas sem que esta afete diretamente a intenção de ajudar. Apesar disso, tais atribuições e reações acabam impactando o acesso a cuidados de saúde de pessoas gays e trans devido a processos sutis de exclusão e discriminação. Além disso, no cenário "sexo desprotegido" ocorreram maiores atribuições de controle, culpa e responsabilidade para o homem homossexual e para a mulher trans, indicando que há um viés negativo de atribuição para indivíduos gays e trans.

Levando em consideração os dados que foram apresentados neste estudo, conclui-se que ainda há muito que se avançar em termos de superação de preconceito e estigma relacionado ao HIV/AIDS no Brasil. A maior atribuição de culpa, controle e responsabilidade aos homens homossexuais e às mulheres trans também indica que estamos longe de alcançar a pacificação dos preconceitos contra a diversidade sexual e de gênero. Ademais, a presença de preconceito que foi detectada nesta pesquisa ocorreu entre profissionais e estudantes da área da saúde, um segmento específico da população. Possivelmente, se estudos como esse fossem ampliados para a população geral, talvez os dados fossem ainda mais alarmantes.

Portanto, de forma que possa ocorrer a superação dos preconceitos relacionados à orientação sexual e à identidade de gênero, bem como a eliminação do estigma relacionado ao HIV/AIDS, sugere-se que os cursos de graduação das áreas da saúde incluam em seus currículos a temática de HIV/AIDS, bem como a temática de diversidade sexual e de gênero. Ainda, são necessárias políticas públicas e ações de Estado que venham a reduzir o estigma e o preconceito, para que possamos ter uma melhora nesse cenário.

\section{Referências}

Allport, G. (1954). The nature of prejudice. Reading: Addison-Wesley.

Ayres, J. R. C. M., Paiva, V., \& França Junior, I. (2011). From natural history of disease to vulnerability: Changing concepts and practices in contemporary public health. In R. Parker, \& M. Sommer (Eds.), Routledge Handbook in Global Public Health (pp. 98108). New York: Routledge.

Baral, S. D., Poteat, T., Stromdahl, S., Wirtz, A. L., Guadamuz, T. E., \& Beyrer, C. (2013). Worldwide burden of HIV in transgender women: A systematic review and meta- 
Fernando Martins de Azevedo, Damião Soares de Almeida Segundo, Marina Feijó, Henrique Caetano Nardi, Angelo Brandelli Costa

analysis. Lancet Infectious Diseases, 13(3), 214-222. doi:10.1016/S14733099(12)70315-8

Bockting, W., Robinson, B., Benner, A., \& Scheltema, K. (2004). Patient satisfaction with transgender health services. Journal of Sex \& Marital Therapy, 30(4), 277-294. doi:10.1080/00926230490422467

Cobb, M., \& Chabert, J. (2002). HIV/AIDS and care provider attributions: Who's to blame?. AIDS Care, 14(4). 545-548. doi:10.1080/09540120208629672

Costa, A. B. (2015). Vulnerabilidade para o HIV em mulheres trans: O papel da psicologia e o acesso à saúde. (Tese de Doutorado). Universidade Federal do Rio Grande do Sul, Porto Alegre, RS, Brasil. Recuperado de http://www.lume.ufrgs.br/handle/10183/140827

Costa, A. B., Fontanari, A. M. V., Jacinto, M. M., Silva, D. C., Lorencetti, E. K., Rosa Filho, H. T., . . \& \& Lobato, M. I. R. (2015). Population-based HIV prevalence and associated factors in male-to-female transsexuals from Southern Brazil. Archives of Sexual Behavior, 44(2), 521-524. doi:10.1007/s10508-014-0386-Z

Costa, A. B., Fontanari, A. M. V., Catelan, R. F., Schwarz, K., Stucky, J. L., Rosa Filho, H. T., Pase, P. F., Gagliotti, D. A. M., Saadeh, A., Lobato, M. I. R., Nardi, H. C., Koller, S. H. (2018). HIV-Related Healthcare Needs and Access Barriers for Brazilian Transgender and Gender Diverse People. AIDS and Behavior, 22(8), 2534-2542. doi:10.1007/s10461-017-2021-1

Dela Coleta, J. A., \& Dela Coleta, M. F. (2006). Atribuição de causalidade: Teoria, pesquisa e aplicações. Taubaté: Cabral Livraria e Editora Universitária.

Dooley, P. A. (1995). Perceptions of the Onset Controllability of AIDS and Helping Judgments: An Attributional Analysis. Journal Applied Social Psychology, 25(10), 858-869. doi:10.1111/j.1559-1816.1995.tb02649.x

French, D. P., Senior, V., Weinman, J., \& Marteau, T. M. (2001). Causal attributions for heart disease: A systematic review. Psychology and Health, 16(1), 77-98. doi: $10.1080 / 08870440108405491$

Hamman, C., Pizzinato, A., Weber, J. L. A., \& Rocha, K. B. (2017). Narrativas sobre risco e culpa entre usuários e usuárias de um serviço especializado em infecções por HIV: Implicações para o cuidado em saúde sexual. Saúde e Sociedade, 26(3), 651-663. doi:10.1590/s0104-12902017170669

Heider, F. (1970). The psychology of interpersonal relations. New York: Wiley. 
Fernando Martins de Azevedo, Damião Soares de Almeida Segundo, Marina Feijó, Henrique Caetano Nardi, Angelo Brandelli Costa

Katz, I. T., Ryu, A. E., Onuegbu, A. G., Psaros, C., Weiser, S. D., Bangsberg, D. R., \& Tsai, A. C. (2013). Impact of HIV-related stigma on treatment adherence: Systematic review and meta-synthesis. Journal of the International AIDS Society, 16, 18640. doi:10.7448/IAS.16.3.18640

Mantler, J., Schellenberg, E. G., \& Page, J. S. (2003). Attributions for serious illness: Are controllability, responsibility and blame different constructs? Canadian Journal of Behavioural Science, 35(2), 142-152. doi:10.1037/h0087196

Matos, I. B., Toassi, R. F. C., \& de Oliveira, M. C. (2013). Profissões e ocupações de saúde e o processo de feminização: Tendências e implicações. Athenea digital, 13(2), 239-244.

McDonell, J. R. (1993). Judgments of personal responsibility for HIV infection: An attributional analysis. Social Work, 38(4), 403-410. doi:10.1093/sw/38.4.403

Melo, L. N. (2006). Perfil de comportamento de risco para HIV/DST em homens que fazem sexo com homens (HSH) a partir do uso da técnica de amostragem Time Space Sampling (TSS) (Dissertação de Mestrado). Universidade Federal do Rio Grande do Sul, Porto Alegre, RS, Brasil. Recuperado de http://www.lume.ufrgs.br/handle/10183/26124

Ministério da Saúde. (2015). Boletim Epidemiológico AIDS - DST. Brasília: Autor.

Ministério da Saúde. (2018). Boletim Epidemiológico HIV/AIDS. Brasília: Autor.

Meyer, I. H. (1995). Minority stress and mental health in gay men. Journal of Health and Social Behavior, 36(1), 38-56. doi:10.2307/2137286

Nasser, R., Singhal, S., \& Abouchedid, K. (2005). Causal attributions for poverty among Indian youth. Current Research in Social Psychology, 11(1), 1-13. doi:10.30849/rip/ijp.v51i1.109

Poteat, T., Wirtz, A., Radix, A., Borquez, A., Silva-Santisteban, A., . . . \& Deutsch, M. (2015). HIV risk and preventive interventions in transgender women sex workers. The Lancet, 385(9964), 274-286. doi:10.1016/s0140-6736(14)60833-3

Rueda, S., Mitra, S., Chen, S., Gogolishvili, D., Globerman, J., Chambers, L., ... \& Rourke, S. B. (2016). Examining the associations between HIV-related stigma and health outcomes in people living with HIV/AIDS: A series of meta-analyses. BMJ Open, 6(7), e011453. doi:10.1136/bmjopen-2016-011453

Safer, J. D., Coleman, E., Feldman, J., Garofalo, R., Hembree, W., Radix, A., \& Sevelius, J. (2016). Barriers to health care for transgender individuals. Current Opinion in Endocrinology, Diabetes and Obesity, 23(2), 168-171. doi:10.1097/MED.0000000000000227 
Fernando Martins de Azevedo, Damião Soares de Almeida Segundo, Marina Feijó, Henrique Caetano Nardi, Angelo Brandelli Costa

Seacat, J. D., Hirschman, R., \& Mickelson, K. D. (2007). Attributions of HIV onset controllability, emotional reactions, and helping intentions: Implicit effects of victim sexual orientation. Journal of Applied Social Psychology, 37(7), 1442-1461. doi:10.1111/j.1559-1816.2007.00220.x

Secretaria Municipal de Saúde de Porto Alegre. (2012). Boletim Epidemiológico. Porto Alegre: Autor. Recuperado de http://lproweb.procempa.com.br/pmpa/prefpoa/cgvs/usu_doc/boletim_48_maio_2012versao_final.pdf

Sikorski, C., Luppa, M., Kaiser, M., Glaesmer, H., Schomerus, G., König, H. H., \& RiedelHeller, S. G. (2011). The stigma of obesity in the general public and its implications for public health-a systematic review. BMC public health, 11(1), 661. doi:10.1186/1471-2458-11-661

Weiner, B. (1995). Judgments of responsibility. New York: Guilford Press.

\section{Endereço para correspondência}

\section{Fernando Martins de Azevedo}

Rua Anita Garibaldi, 1143 conjunto 407, Mont'Serrat, Porto Alegre - RS, Brasil. CEP 90450-001

Endereço eletrônico: fernandoazevedo940@gmail.com

\section{Damião Soares de Almeida Segundo}

Rua Ramiro Barcelos, 2600 sala 204, Santa Cecilia, Porto Alegre - RS, Brasil. CEP 90035-003

Endereço eletrônico: damiao_soares@ hotmail.com

\section{Marina Feijó}

Rua da República, 626 apto 404, Cidade Baixa, Porto Alegre - RS, Brasil. CEP 90050-320

Endereço eletrônico: marina.feijo@edu.pucrs.br

\section{Henrique Caetano Nardi}

Rua Ramiro Barcelos, 2600 sala 204, Santa Cecilia, Porto Alegre - RS, Brasil. CEP 90035-003

Endereço eletrônico: hcnardi@gmail.com

\section{Angelo Brandelli Costa}

Avenida Ipiranga, 6681 Prédio 11 sala 933, Partenon, Porto Alegre - RS, Brasil. CEP 90619-900

Endereço eletrônico: angelo.costa@pucrs.br

Recebido em: 26/07/2019

Reformulado em: 09/04/2020

Aceito em: 09/05/2020

Notas 
Fernando Martins de Azevedo, Damião Soares de Almeida Segundo, Marina Feijó, Henrique Caetano Nardi, Angelo Brandelli Costa

* Doutorando em Psicologia pela Universidade Federal do Rio Grande do Sul, UFRGS.

** Doutorando em Psicologia pela Universidade Federal do Rio Grande do Sul, UFRGS.

*** Graduanda do curso de Psicologia da Pontifícia Universidade Católica do Rio Grande do Sul, PUCRS.

**** Professor do Programa de Pós-Graduação em Psicologia Social e Institucional da Universidade Federal do Rio Grande do Sul, UFRGS.

***** Professor do Programa de Pós-Graduação em Psicologia da Pontifícia Universidade Católica do Rio Grande do Sul, PUCRS.

Este artigo de revista Estudos e Pesquisas em Psicologia é licenciado sob uma Licença Creative Commons Atribuição-Não Comercial 3.0 Não Adaptada. 